ESJ Humanities

\title{
Gestion des déchets ménagers dans le quartier Mambanda (Douala-Cameroun) : Quelles stratégies durables?
}

\author{
Mougoue Benoût
}

Maîtres de conférences, Département de Géographie, Faculté des Arts,

Lettres et Sciences Humaines, Université de Yaoundé I

Agofak Clarisse Viviane

Doctorante en Géographie, Département de Géographie, Faculté des Arts,

Lettres et Sciences Humaines, Université de Yaoundé I

Nya Esther Laurentine

Doctorat $\mathrm{PhD}$, assistant, Département de Géographie, Faculté des Arts, Lettres et Sciences Humaines, Université de Maroua

Doi:10.19044/esj.2021.v17n39p138

Submitted: 29 March 2021

Accepted: 29 November 2021

Published: 30 November 2021
Copyright 2021 Author(s)

Under Creative Commons BY-NC-ND

4.0 OPEN ACCESS

Cite As:

Benoit M., Viviane A.C. \& Laurentine N.E.(2021). Gestion des déchets ménagers dans le quartier Mambanda (Douala-Cameroun) : Quelles stratégies durables?. European Scientific Journal, ESJ, 17 (39), 138. https://doi.org/10.19044/esj.2021.v17n39p138

\section{Resumé}

L'urbanisation est l'une des grandes caractéristiques du monde contemporain. Elle génère des effets sur l'environnement et le cadre de vie perceptibles à travers la gestion des déchets. En effet, l'urbanisation s'accompagne d'une croissance de la population urbaine qui à son tour induit une production sans cesse importante de déchets, souvent mal gérés. Cette réflexion vise à cerner les problèmes liés aux différentes pratiques de gestion des déchets ménagers dans le quartier Mambanda de la ville de Douala. L'étude s'appuie sur une approche à la fois qualitative et quantitative qui intègre les recherches documentaires, les observations de terrain et les entretiens avec les acteurs-clé du terrain. Les résultats de l'étude montrent que les déchets solides, liquides et gazeux produits dans le quartier Mambanda sont constitués essentiellement des restes de nourriture, d'eaux usées domestiques et résiduaires, des eaux vannes, des déjections humaines et animales... Les populations, l'Etat et les sociétés privées (Hysacam, CIPRE, associations...) sont les acteurs qui se déploient sur le terrain pour gérer ces déchets qui sont généralement déversés dans les rues, les caniveaux, les cours 
d'eaux, les champs, les drains et les bacs à ordures. La mauvaise gestion des déchets entraine, dans la plupart des cas, la dégradation de l'environnement et du cadre de vie des populations et par conséquent impacte la santé des populations qui y vivent. Ainsi, la forte implication des populations dans le processus de gestion des déchets (campagnes d'éducation à l'hygiène, création et entretien du réseau d'évacuation...) est la meilleure stratégie durable pour une gestion efficace de ces déchets.

Mots cles: Déchets ménagers, Douala, gestion, insalubrité, risques sanitaires

\title{
Household Waste Manage Management in Mambanda (Douala-Cameroun): What Strategies to Sustainable?
}

\section{Mougoue Benoût}

Maîtres de conférences, Département de Géographie, Faculté des Arts, Lettres et Sciences Humaines, Université de Yaoundé I

\section{Agofak Clarisse Viviane}

Doctorante en Géographie, Département de Géographie, Faculté des Arts, Lettres et Sciences Humaines, Université de Yaoundé I

\section{Nya Esther Laurentine}

Doctorat $\mathrm{PhD}$, assistant, Département de Géographie, Faculté des Arts, Lettres et Sciences Humaines, Université de Maroua

\begin{abstract}
Urbanization is one of the great characteristics of the contemporary world. It generates perceptible effects on the environment and the living environment through waste management. Indeed, urbanization is accompanied by a growth of the urban population which in turn induces an ever-increasing production of waste, which is often poorly managed. This reflection aims to identify the problems related to the different household waste management practices in the Mambanda district of the city of Douala. The study is based on a qualitative and quantitative approach that integrates documentary research, observations and interviews with key players in the field.The results of the study show that the solid, liquid and gaseous waste produced in the Mambanda district consists mainly of food scraps, domestic and waste water, human and animal excreta. The populations, the State and private companies (Hysacam, CIPRE, associations, etc.) are the actors who are deployed in the field to manage this waste which is generally dumped in the streets, gutters, rivers, fields., drains and garbage bins. Poor waste management leads, in most cases, to the degradation of the environment and the living environment of the populations. Thus, the strong involvement of the population in the waste management process (hygiene education
\end{abstract}


campaigns, creation and maintenance of the disposal network, etc.) is the best sustainable strategy for effective waste management.

Keywords: Household waste, Douala, management, insalubrity, health risks

\section{Introduction}

Les grandes villes africaines, les capitales d'Etat en particulier, ont connu une croissance démographique rapide au cours des quatre dernières décennies (Adamah, 2001). Cette croissance exponentielle pose aux gestionnaires urbains, de grands défis notamment dans le domaine de l'assainissement, de la collecte et du traitement des déchets.

La population urbaine mondiale était de 3,8 milliards d'habitants en 2013, un chiffre qui devrait monter en flèche pour s'établir à près de 6,3 milliards en 2050 (Banque Mondiale, 2004). Cette explosion de la population urbaine s'accompagne de l'augmentation de la production des déchets, difficilement gérés par les ménages urbains dans les pays en développement. Cette réalité est très visible dans les quartiers précaires des villes d'Afrique (Sane, 2002). Edifiés sans planification, les populations accusent un manque criard de système d'assainissement. Dans l'ensemble, la gestion des déchets solides et des eaux usées est un problème de gouvernance urbaine pour tous les Etats Africains en général et l'Etat camerounais en particulier (Paul, 2001).

Douala, capitale économique du Cameroun, n'échappe pas à cette réalité. En effet, cette ville possède de nombreux atouts économiques (port, aéroport, industries, etc.) qui contribuent énormément au développement des transactions commerciales sous régionales et même internationales. Il s'en suit une forte pression démographique engendrée à la fois par un taux de natalité élevé (4\%), un important déplacement des populations de l'arrière-pays vers la métropole et un afflux d'immigrants (6\%) toujours croissant qui pose de multiples problèmes dont la gestion des déchets ménagers (Moutila, 2011). Ce problème se pose avec acuité dans le quartier Mambanda où la gestion des déchets est devenue une réelle menace pour l'environnement et la santé des populations.

Malgré toutes les actions d'assainissement mises en place par les populations, les pouvoirs publics et l'appui du concessionnaire Hysacam et des ONG, l'insalubrité persiste et devient de plus en plus préoccupante. Dans ce quartier, très peu de déchets produits quotidiennement sont évacués (investigations de terrain, 2019). C'est pourquoi, les déchets sont déposés sur la voie publique, les espaces vagues, les lieux illégaux, les cours d'eau, les égouts ou entassées dans des décharges à ciel ouvert. Ce constat amène à s'interroger sur les conséquences des pratiques de gestion de ces déchets. 
Cette étude vise à analyser les différentes stratégies de gestion des déchets ménagers à Mambanda tout en ressortant les problèmes qui y sont liés afin de proposer des stratégies pour leur gestion durable.

\section{Méthodologie}

\subsection{Milieu de l'étude}

Mambanda est le plus vaste quartier de la Commune d'Arrondissement de Douala IV. Il est situé sur les rives du fleuve Wouri, à l'Est de la zone industrielle de Bonabéri, et au Sud de la route nationale $n^{\circ} 3$. Mambanda s'étends sur environ 500 ha, subdivisés en 59 blocs. Sa population est estimée à 70865 habitants ${ }^{1}$. La croissance de la population de Mambanda s'accompagne d'une production accrue des déchets ménagers qui dégradent l'environnement et impactent la santé de cette population.

Figure 1: Localisation de la zone d'étude

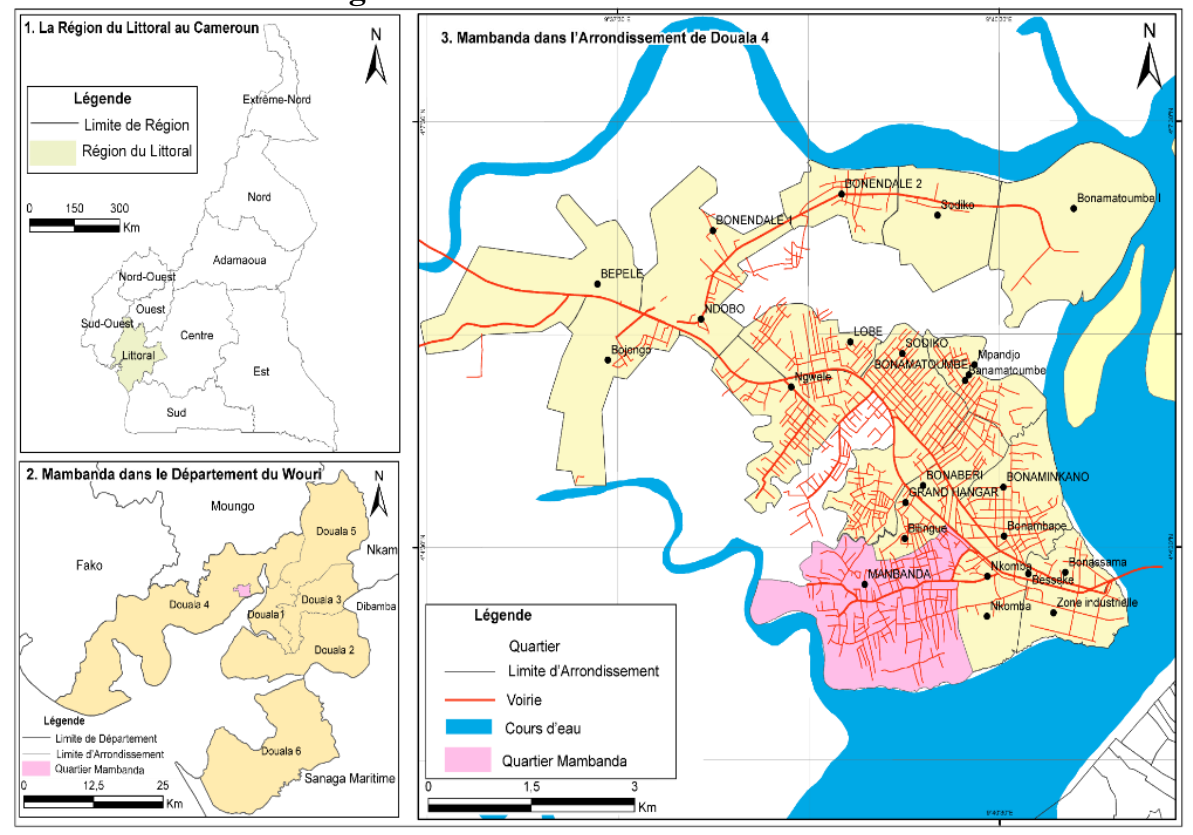

Source: INC, 2014 et investigations de terrain, 2020

\subsection{Collecte des données}

Les données collectées en vue de l'élaboration de cette réflexion sont de deux sources: secondaires et primaires. Cette démarche s'appuie sur l'exploitation des documents, les observations de terrain, les entretiens et l'enquête-ménage. A cet effet, des guides d'entretien et un questionnaire ont été utilisés pour la collecte des données de sources primaires. Ces guides d'entretien et questionnaires s'articulaient autour des rubriques telles que les

${ }^{1}$ BUCREP, 2010 et investigations de terrain, 2018. 
différentes méthodes de gestion de déchets, le lieu de déversement des déchets, les acteurs de la collecte... En raison du nombre important de ménages résidents, un échantillonnage aléatoire de ménages a été opéré dans ce quartier en appliquant le tirage systématique de 2 ménages sur 100 . C'est ainsi que sur 17750 ménages, 355 ont été enquêtés.

Après la collecte des informations, a suivi la phase de dépouillement, du traitement, et de l'interprétation des résultats. A cet effet, le logiciel Word a été utilisé pour la saisie et le traitement de texte, Excel et SPSS pour le dépouillement des questionnaires et la confection des tableaux et figures et les logiciels de cartographie ARC Map 10, Adobe et Illustrator pour l'élaboration des cartes. Le passage de l'enquêteur au sein des ménages se faisait très tôt le matin ou très tard en soirée pour éviter l'exclusion d'une partie de la population cible.

La méthode d'analyse compréhensive et systémique a été mobilisée dans le cadre de cette réflexion. Elle a permis d'analyser les différentes stratégies de gestion des déchets mises en place par les acteurs ainsi que les conséquences induites. Au-delà, elle a permis de mieux saisir les différentes modalités de gestion des déchets dans le quartier Mambanda. L'approche systémique a permis d'analyser l'ensemble des problèmes liés à la gestion des déchets dans ce quartier en vue de planifier des stratégies adéquates de leur éradication. L'analyse systémique a servi à cerner le fonctionnement du système de gestion locale des déchets ménagers dans ce quartier.

\section{Résultats}

3.1. Mambanda : un quartier caractérisé par divers types de déchets

La Commune d'Arrondissement de Douala IV, porte d'entrée et de sortie de la ville de Douala, abrite des établissements industriels et des unités socio-économiques importants. Ces atouts rendent cette commune attrayante et par conséquent, engendrent la croissance rapide de sa population. L'accroissement de cette population s'accompagne de l'augmentation de la production des déchets qui s'élève journalièrement à environ $42519 \mathrm{~kg}^{2}$. Les ménages de Mambanda produisent des déchets de divers types: déchets biodégradables et dégradables, déchets liquides et gazeux.

\section{Déchets biodégradables et dégradables}

Il s'agit ici des déchets solides. A Mambanda, on distingue deux types de déchets solides produits par les ménages. Il s'agit:

- des déchets issus des activités intra-urbaines qui renvoient aux déchets de l'agriculture et de l'élevage. Ces déchets comprennent les éléments

\footnotetext{
${ }^{2}$ Estimations de terrain, 2020. Selon une étude faite par l'ENSP de Yaoundé, chaque
} habitant produit en moyen $600 \mathrm{~g}$ de déchets par jour 
de la litière ou de la nécro masse, les résidus des cultures, les avaries de produits issus des récoltes ;

- des déchets ménagers proprement dits comprenant tout ce qui doit être jeté ou entassé dans la nature, tout ce dont l'homme doit s'en débarrasser provenant des habitations ou des logements ( avaries de nourriture, plastiques, vielles boites de conserve, vieux vêtements, vielles chaussures...). Selon Ngnikam et Tanawa (2006), les déchets ménagers sont aussi des déchets liés à une activité occasionnelle qui, en raison de leur volume et de leur poids, ne peuvent être pris en compte par la collecte régulière des ordures ménagères et les déchets présentant un ou plusieurs caractères dommageables pour l'environnement et/ou qui ne peuvent pas être éliminés par les mêmes voies que les ordures ménagères sans créer des risques lors de la collecte. Lorsque ces déchets sont mal gérés, les nuisances et les pollutions apparaissent.

\section{Déchets liquides}

Les habitants de Mambanda produisent quotidiennement d'importantes quantités de déchets liquides constitués essentiellement de graisses et des huiles issues des différentes activités de cuisson, des eaux usées provenant de la lessive et des travaux ménagers (nettoyage des toilettes, du logement, de la vaisselle...), des eaux vannes provenant des fosses septiques mal construites et mal entretenues qui laissent les urines et les eaux de bain s'écouler dans la nature, et du nettoyage des enclos d'élevage. A Mambanda, les eaux usées proviennent de trois principales sources (figure 2).

Figure 2 : Répartition des ménages en fonction de l'origine des déchets liquides

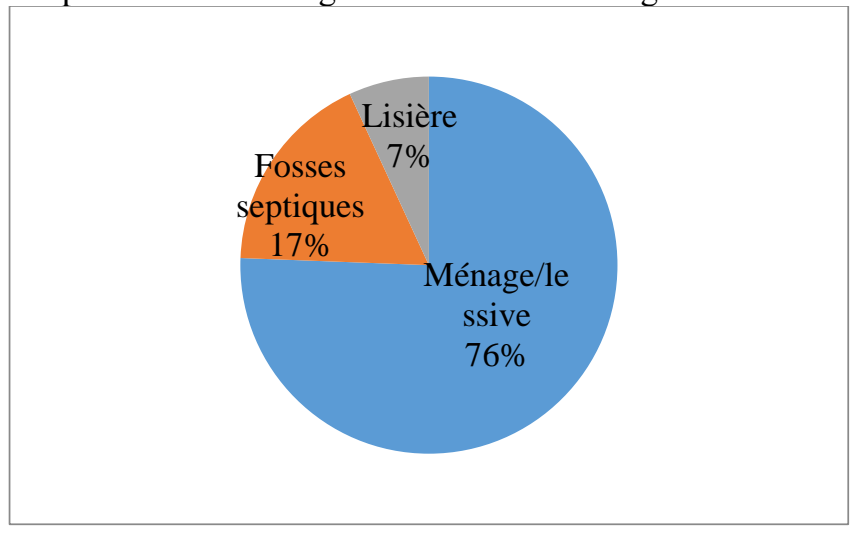

Source: Investigations de terrain, 2019.

$76 \%$ des ménages de Mambanda affirment que leurs eaux usées proviennent essentiellement des activités de ménage et de la lessive. 17\% pensent que les fosses septiques produisent d'énormes quantités de déchets 
liquides et $6 \%$ de ménages, estiment que ces déchets proviennent du nettoyage des enclos d'élevage.

\section{Déchets gazeux}

Il s'agit de toute substance gazeuse libérée par l'incinération d'un objet ou par une réaction chimique quelconque qui peut conduire à une nuisance ou pollution. Ces déchets sont le plus souvent des gaz invisibles comme le monoxyde de carbone (Co), les oxydes d'azote (No), et de soufre (So2), ou encore l'ozone (O3), des particules solides, plus ou moins fines. Elles donnent leurs consistances et couleurs aux fumées. A Mambanda, les déchets gazeux proviennent essentiellement de l'utilisation du bois de chauffe et du charbon pour la cuisson, de l'incinération des déchets, des fumées de cigarette, des déchets de jardins ou vieux vêtements, des émissions des gazs issus des décharges des déchets solides en décomposition, des systèmes de traitement des eaux ménagers et de la poussière. Ainsi, les populations de Mambanda énumèrent la consommation des cigarettes, l'incinération des déchets et l'utilisation du bois de chauffe pour la cuisson comme étant les principales sources de production des déchets gazeux dans leur milieu de vie (figure 3).

Figure 3 : Répartition des ménages en fonction des sources de production des déchets gazeux

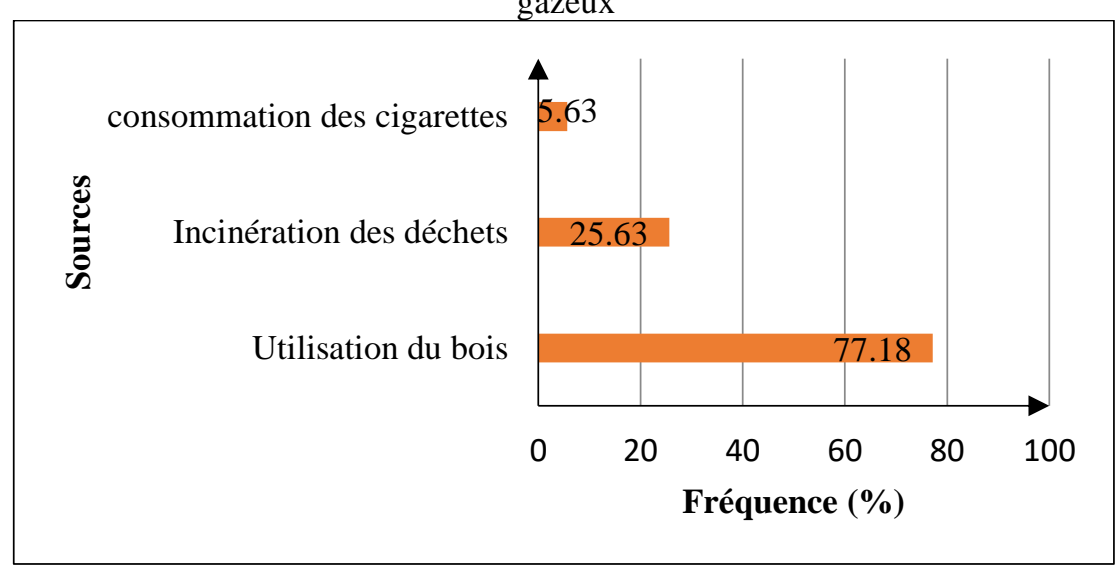

Source: Investigations de terrain, 2019.

Selon les populations de Mambanda, 77,18\% de déchets gazeux proviennent de l'utilisation du bois de chauffe et du charbon, 25,63\% de l'incinération des ordures et 5,63\% de la consommation des cigarettes.

\subsection{Stratégies de gestion des déchets à Mambanda}

Généralement, la gestion des déchets incombe au Ministère en charge de l'environnement et de l'écologie. Cependant, au Cameroun, beaucoup d'autres acteurs sont impliqués dans la filière de gestion des déchets. Il s'agit : 
- des acteurs institutionnels (Ministères, Communes, Institutions de recherche). On distingue deux grandes catégories à savoir les institutions de planification, d'orientation et de contrôle (MINEP, MINADEM, MINEE, MINATD, MINHDU, MINDUH, MINSANTE, MINNIMIDT) et les Collectivités Territoriales Décentralisées (CTD).

- des acteurs financiers et techniques. Il s'agit ici du Ministère des Finances et du Fonds Spécial d'Equipement et d'Intervention Intercommunale (FEICOM),

- des acteurs informels que sont les Organismes Non Gouvernementaux qui participent à la collecte et/ou au traitement des déchets, aux populations locales et aux sociétés privées.

A Mambanda, ces acteurs sont presque tous présents et contribuent d'une façon ou d'une autre à la gestion des déchets ménagers.

\section{Population : Acteur clé de la gestion des déchets ménagers à Mambanda}

A Mambanda, la population est un acteur influant de la gestion des déchets. Cependant, faute de moyens adéquats ou à cause de l'incivisme, les déchets s'accumulent dans l'espace urbain (photo 1). Les populations déversent leurs déchets dans la rue, autour de leurs logements, dans les rigoles, sur les espaces non bâtis (cf. tableau 1).

Dans ce quartier, les rues sont caractérisées par d'importants dépôts d'ordures qui enlaidissent le paysage. C'est l'exemple de la rue qui mène au grand stade de Mambanda (photo1).

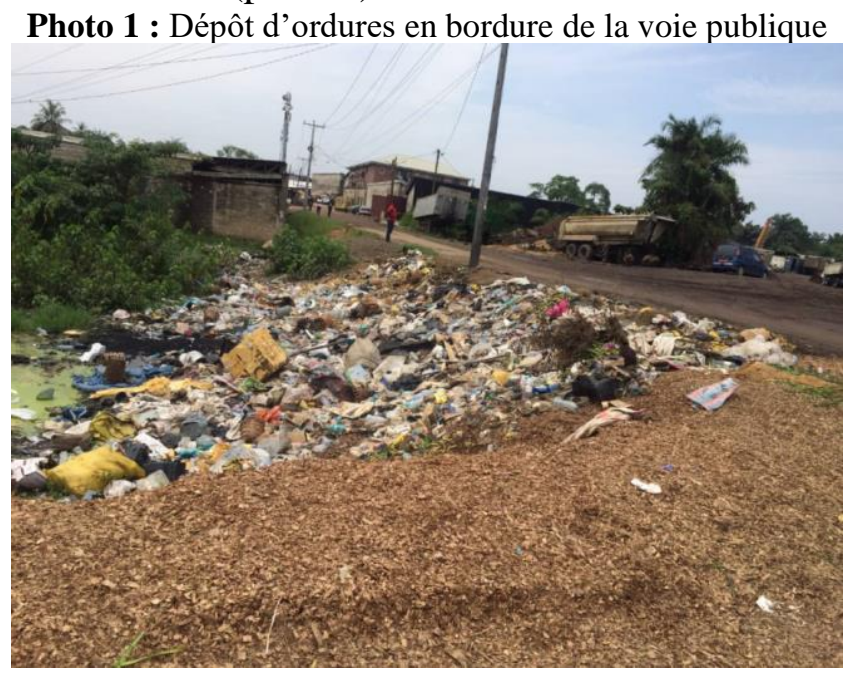

Source : Agofak 2019

Cette photo montre une décharge à Mambanda. Les populations ont transformé les abords de cette rue en dépotoir d'ordures de telle sorte qu'elle a été rétrécie et le paysage enlaidi. 
Les déchets déversés sur les terrains vagues, le long des voies, dans les cours d'eau, les caniveaux, encombrent les drains, les égouts, les voies publiques. Ces déchets, mal gérés, freinent la circulation automobile. Les populations sont parfois débordées par la quantité de déchets à éliminer, ce qui induit l'incinération d'une bonne partie dans les foyers.

Tableau 1 : Lieu de déversement des déchets solides et liquides

\begin{tabular}{|c|r|r|r|r|r|r|r|}
\hline & $\begin{array}{c}\text { Dans la } \\
\text { cours }\end{array}$ & $\begin{array}{c}\text { Drains ou } \\
\text { rigoles }\end{array}$ & Rues & \multicolumn{1}{c|}{$\begin{array}{c}\text { Bacs à } \\
\text { ordures }\end{array}$} & Trous & Champs & $\begin{array}{c}\text { Cours } \\
\text { d'eau }\end{array}$ \\
\hline $\begin{array}{c}\text { Déchets } \\
\text { liquides }\end{array}$ & 3,09 & 24,78 & 1,40 & 00 & 2,53 & 59,71 & 8,45 \\
\hline $\begin{array}{c}\text { Déchets } \\
\text { solides }\end{array}$ & 5,35 & 3,38 & 13,80 & 13,23 & 4,50 & 47,04 & 12,67 \\
\hline
\end{tabular}

Source: Investigations de terrain, 2019.

Les investigations de terrain révèlent que $59,71 \%$ de ménages déversent leurs déchets liquides dans les champs et $47,04 \%$ y évacuent leurs déchets solides. Cependant, certains habitants de Mambanda incinèrent leurs déchets et déversent les cendres dans les caniveaux. D'autres, après incinération, jettent les cendres dans des décharges à ciel ouvert, souvent non autorisées.

Ainsi, l'intensification des activités marchandes dans le quartier a entrainé l'occupation de tout l'espace disponible. Les petits commerçants bloquent les voies d'accès et enfreignent les règlements de recul. En plus de l'augmentation de la production de déchets, cette situation entrave les mécanismes de collecte des rebuts.

Contrairement à d'autres quartiers de Douala et de la Commune d'Arrondissement de Douala IV, Mambanda est traversé par un grand cours d'eau où certains ménages y déversent leurs ordures. Cette pratique est presque inexistante dans d'autres quartiers de Douala.

\section{Etat : l'un des piliers de la gestion des déchets ménagers}

A Mambanda, l'Etat, à travers la Commune d'Arrondissement de Douala IV et la Communauté Urbaine de Douala, mène des activités de propreté dans les espaces marchands et le long des voies publiques. L'opération «zéro tas d'ordures » initiée dans les écoles par la Communauté Urbaine de Douala est l'une des initiatives de gestion des déchets dans ce quartier. Allant dans le même sens, l'opération « Mercredi propre », initiée par les autorités communales, consiste à la descente sur le terrain des agents de la Mairie chaque mercredi en vue de balayer les rues, de nettoyer les caniveaux et les rigoles (photo 2). Ainsi, chaque mercredi, les agents de la Commune d'Arrondissement de Douala IV, se déploient sur le terrain afin d'assainir l'environnement (cf.photo 3). e rendre salubre le paysage comme il est constaté sur la photo ci-dessous. 
Photo 2 : Opération « Mercredi propre » par les agents de la Commune de Douala IV

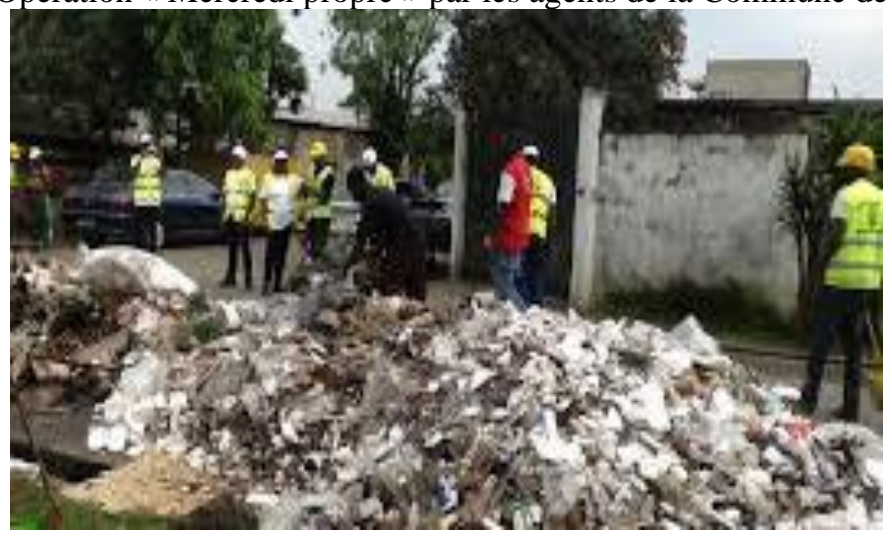

Source : Agofak, 2019

Cette photo montre les agents de la Commune d'Arrondissement de Douala IV (chasuble vert -citron) en plein investissement humain. Ces agents ont pour devoir de nettoyer les rues, curer les rigoles et caniveaux, désherber les lieux publics envahis par les mauvaises herbes.

Toujours, dans l'optique d'assainissement et de protection de l'environnement, la Communauté Urbaine de Douala (CUD) a mis à la disposition des populations, des camions de transport des boues de vidange et des boues industrielles. De même, les camions de transport des déchets assimilables aux ordures ménagères et aux avaries de déchets alimentaires ont été mis en marche et les décharges municipales spécialisées créées. Toutes ces actions contribuent à assainir le milieu.

\section{Sociétés privées et ONG : Acteurs de la gestion des déchets}

Les sociétés privées et les ONG occupent une place de choix dans la gestion des déchets à Mambanda. Il s'agit de Hysacam, du CIPRE, des associations et des pré-collecteurs.

\section{- Concessionnaire}

Hysacam œuvre dans la gestion des déchets à Mambanda. Elle dispose des bacs qui constituent les points de concentration des ordures. Elle assure la collecte, le transport et le traitement des ordures ménagères, le balayage et le nettoyage des principales rues, des places publiques et marchés. Cependant, cette collecte n'est pas régulière car le passage de ces camions n'est toujours pas fréquent. En plus, les bacs sont peu nombreux et mal repartis. Il n'y en a pas assez à chaque dépôt, et lorsqu'ils sont pleins, les résidents déposent leurs déchets à même le sol. La plupart des décharges sont éloignées des utilisateurs cibles. C'est pourquoi, les résidents sont contraints de jeter leurs déchets dans la nature, ou dans des lieux peu indiqués.

Les déchets solides déposés dans les bacs placés en bordure des grandes voies et des marchés sont vidés par les éboueurs de Hysacam (photo 
3) et le contenu transporté vers la grande décharge située à la zone industrielle de Bonabéri.

Photo 3 : Action de la société Hysacam dans le processus de gestion des déchets à

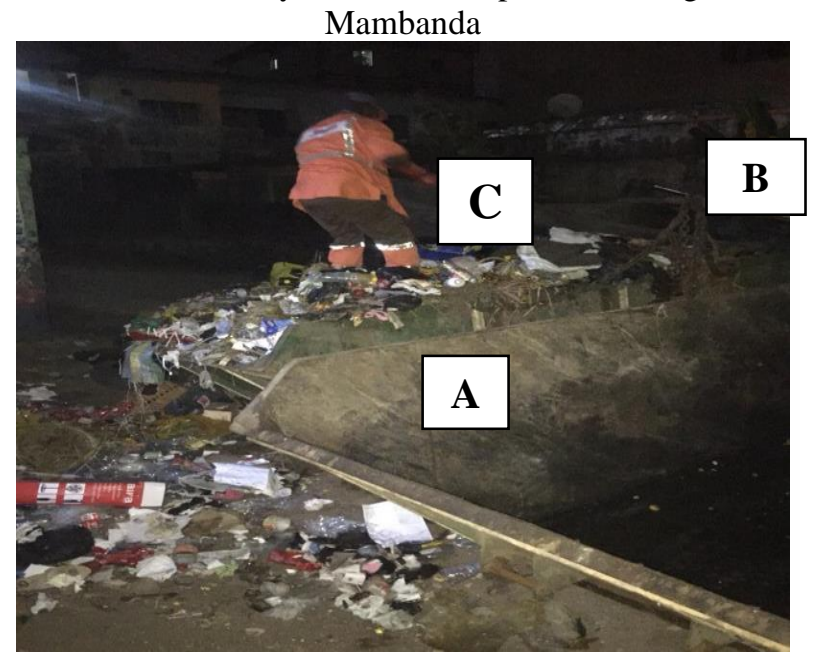

Source : Agofak, 2019.

Cette photo met en relief les bacs à ordures $(\boldsymbol{A})$ disposés par la société Hysacam. L'un des camions de cette société $(\boldsymbol{B})$ a été conduit en ce lieu par quelques éboueurs $(\boldsymbol{C})$ dans le but de transporter les déchets de ces bacs.

Hysacam n'effectue pas la collecte des ordures ménagères de porte-àporte à cause de l'enclavement de la zone. Les résidents doivent s'occuper eux-mêmes de leurs déchets. Lorsque les bacs sont éloignés, notamment dans les zones centrales inaccessibles du quartier, ils sont tentés de s'en débarrasser par des dépôts anarchiques in situ.

Au niveau des points de transit, on a développé l'apport volontaire des déchets. En effet, Hysacam et la population se sont entendus pour créer un point de transit au stade Mambanda où certains résidents de Mambanda y apportent volontairement leurs déchets ce qui n'est pas le cas dans d'autres quartiers de la Commune d'Arrondissement de Douala IV.

\section{- ONG et Associations}

Au-delà d'Hysacam, les $\mathrm{ONG}$ et les associations interviennent dans la gestion des déchets ménagers à Mambanda. C'est l'exemple de l'ONG « Centre International de Promotion de la Récupération (CIPRE) » qui œuvre dans le tri et la transformation des déchets plastiques. Quelques associations ont dans leur plan d'action un volet réservé à la gestion des déchets et à l'assainissement du milieu. Il s'agit des associations telles que le club des jeunes dynamiques de Mambanda, l'association Halte à la pollution... Ces associations œuvrent dans la lutte contre la pollution à travers la gestion des déchets et l'assainissement du milieu. 


\section{- Apport des pré-collecteurs}

A côté des ONG et associations, l'action des pré-collecteurs n'est pas négligeable. En effet, selon les investigations de terrain, 10\% des ménages environ ont recours aux pré-collecteurs dans l'évacuation de leurs déchets. Malgré les coûts de prestation relativement bas des pré-collecteurs d'ordures, bon nombre de ménages ne parviennent pas à s'abonner. Le coût mensuel d'abonnement est fixé entre 1000 et 2000 F CFA, en fonction de la fréquence de la pré-collecte. Ce tarif est aussi variable en fonction des structures. Si les abonnés de l'entreprise « SILO » déboursent mensuellement 1500 à $2000 \mathrm{~F}$ $\mathrm{CFA}$, ceux de «BAWO » et «YILEMDE » ne payent que $1000 \mathrm{~F}$ CFA par mois et cela quelle que soit la fréquence de la pré-collecte. Ces structures de pré-collecte des ordures utilisent des tricycles qui leur permettent de faire du porte-à-porte auprès des clients même quand les voies ne sont pas aménagées. Ces structures comportent des inconvénients dus à leur faible capacité de transport et/au manque de matériel de traitement de ces déchets. Ce qui fait que les ordures collectées par ces entreprises sont déversées dans des espaces non appropriés. Tel est le cas de la décharge publique du grand stade de Mambanda.

\subsection{Problèmes liés à la mauvaise gestion des déchets}

La mauvaise gestion des déchets dans la ville de Douala en général et dans la Commune d'Arrondissement de Douala IV en particulier est source de nombreux problèmes.

\section{Obstruction des caniveaux et fréquence des inondations}

L'obstruction des caniveaux est visible à Mambanda, surtout au niveau des caniveaux à ciel ouvert, en bordure des rues. L'obstruction des caniveaux par les déchets induit l'inondation et l'enclavement du quartier Mambanda pendant la saison pluvieuse. Les caniveaux censés drainer l'eau pluviale sont utilisés comme poubelles. Or, c'est bien connu qu'on n'arrête pas l'eau qui veut ou qui doit s'écouler. Lorsqu'on tente de l'arrêter, elle trouve fatalement une autre issue au point d'envahir parfois des endroits où rien n'est prévu. Pour continuer son écoulement, l'eau est obligée d'envahir des espaces aménagés pour générer des inondations. 
Planche 1 : Conséquences de la mauvaise gestion des déchets.

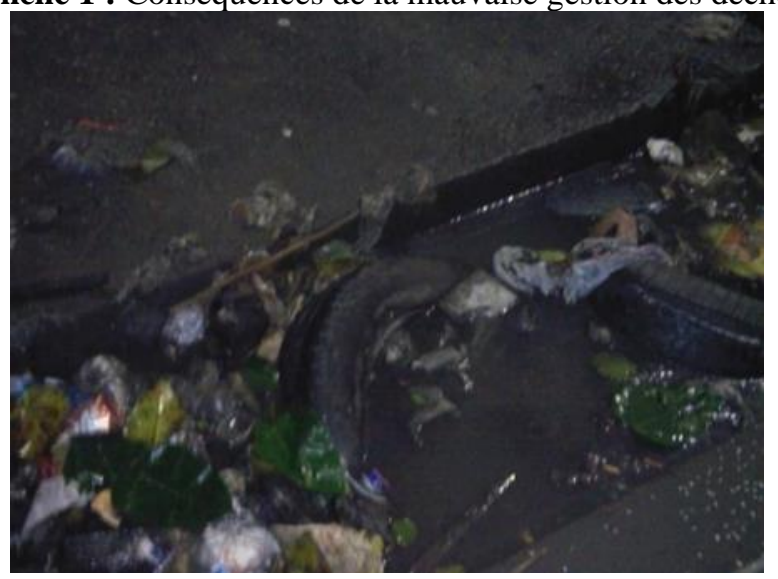

Source : Agofak, 2019

Photo 4 : Un caniveau transformé en poubelle

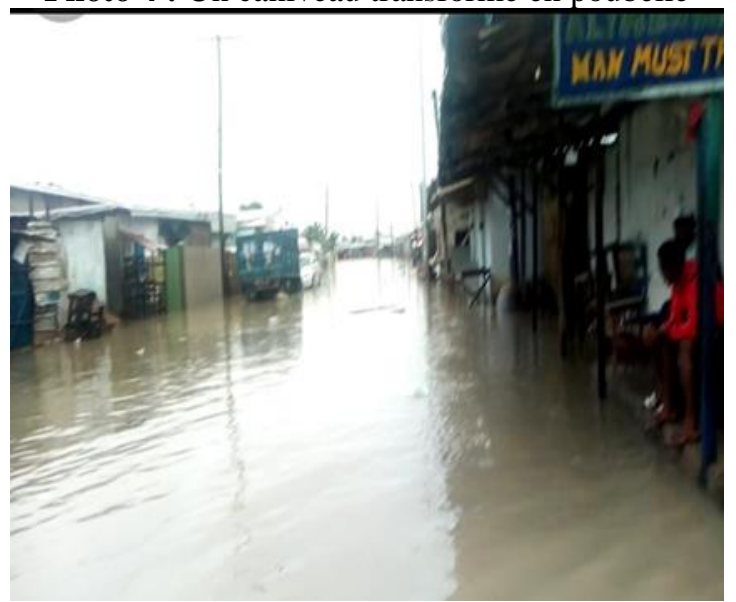

Source : Agofak, 2019

Ce caniveau sur cette photo n'est pas une poubelle mais une infrastructure d'assainissement qui sert de poubelles. Les vieux pneus qui y ont été jetés par les populations obstruent ce caniveau d'où les inondations par endroits. 


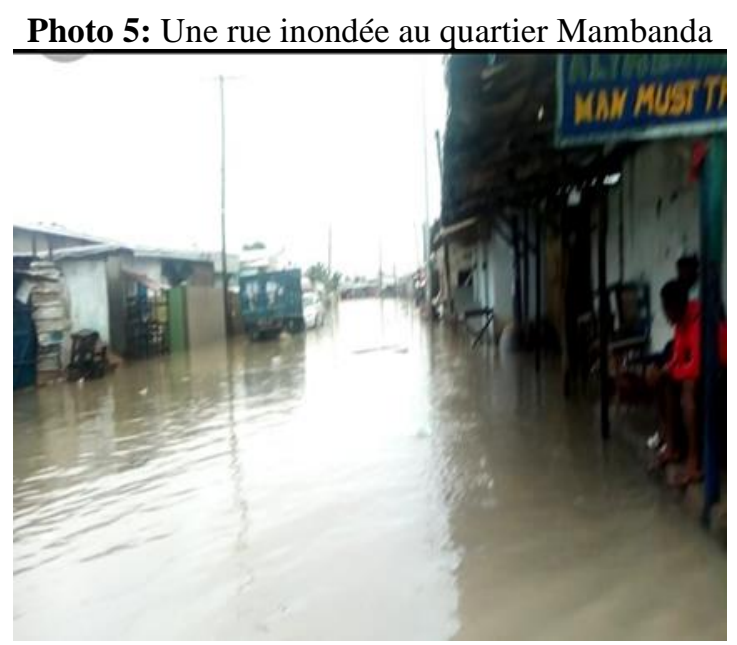

Source : Agofak, 2019

Cette photo présente une rue envahie par les eaux de pluies. Ces inondations sont liées au fait que les caniveaux qui étaient conçus pour drainer les eaux pluviales sont aujourd'hui envahis de déchets. Par conséquence, on note un débordement des eaux en saison de pluies créant ainsi de réels problèmes de stagnation des eaux.

Pendant la saison des pluies, Mambanda se transforme en océan habité par endroits. Aucune artère n'y échappe. Ces inondations sont liées à l'obstruction des canalisations d'eaux par les ordures. Lorsque ce ne sont pas les fameux sacs plastiques qui en sont la cause, c'est carrément les égouts qui sont obstrués par les déchets.

\section{Conflits entre voisins}

A Mambanda, la mauvaise gestion des déchets engendre parfois des conflits entre voisins. Ce sont: les problèmes liés au dépôt des ordures devant ou derrière la maison de son voisin, les conflits dus à l'orientation d'eau de puisard chez le voisin ; les problèmes liés à la glissade grave sur l'eau versée sur la voie par un voisin ; les discordes liées au fait qu'une personne a orienté la toiture de sa maison vers la cour de son voisin, entrainant l'inondation en saison de pluies.

Face à cette multitude de problèmes, on assiste à des querelles, des injures, des bagarres qui sont parfois réglées par le biais du chef de quartier ou même portées devant la juridiction administrative.

\section{Dégradation de l'environnement et impacts sur la santé}

Une mauvaise gestion des déchets dans les villes met en péril l'environnement urbain. En effet, l'accumulation des déchets et leur décomposition sont à l'origine des odeurs nauséabondes, de la pollution des sols, des eaux, de l'air et de l'enlaidissement du paysage urbain. Les eaux 
usées et pluviales qui stagnent dans les caniveaux dégagent également des odeurs pestilentielles. L'incinération des ordures dans les dépotoirs sauvages entraine la pollution de l'air par l'émission des gaz qui se dégagent des fumées. Il y resulte donc une dégradation de l'environnement.

A Mambanda, les ordures ménagères accumulées de manière anarchique dans l'espace urbain engendrent de réels problèmes d'hygiène. Les mouches, les moustiques, des cafards les rongeurs et autres insectes nuisibles y foisonnent. De plus en plus les dépotoirs insalubres y favorisent la multiplication des vecteurs de propagation de maladies infectieuses. Ces bestioles nuisibles propagent des maladies que contractent les êtres humains. A Mambanda, la quasi-totalité des ménages se plaignent de la présence des animaux, vecteurs des maladies au sein de leurs domiciles. Ces populations affirment cohabiter avec les souris, les cafards, les mouches, les moustiques... (cf.figure 4).

Figure 4 : Agents responsables des maladies à Mambanda

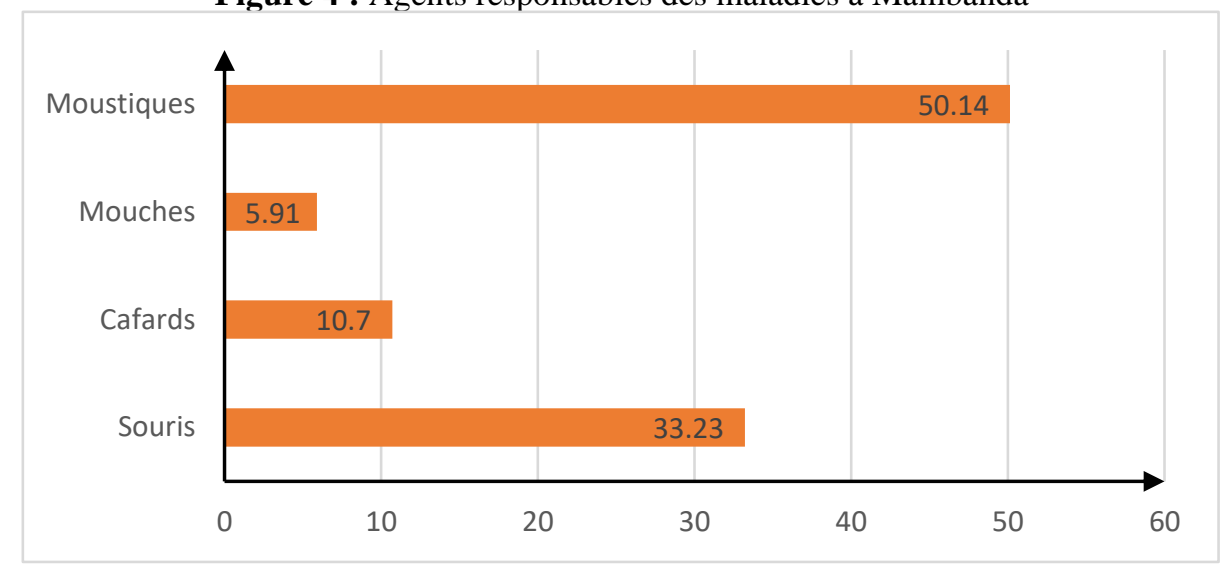

Source: Investigations de terrain, 2019

A Mambanda, 50\% des ménages affirment que leur logement est constamment envahi par des moustiques, 33,23\% cohabitent avec les souris, $10,7 \%$ avec les cafards et $5,91 \%$ avec les mouches. Ces insectes et animaux propagent des maladies au sein de ces logements. C'est ainsi qu'on y note la prolifération des maladies telles que la dysenterie, la diarrhée, le choléra, la fièvre typhoïde et le paludisme, etc.

\subsection{Stratégies durables pour une meilleure gestion des déchets à Mambanda}

Le problème de gestion des déchets se pose avec acuité à Mambanda. Malgré les stratégies mises en place par les populations, l'Etat, les sociétés privées, les ONG et les associations, on y note une insuffisance. Cependant, les populations de Mambanda proposent des stratégies qui selon elles peuvent se révéler efficaces pour la gestion des déchets. Il s'agit de la création des 
comités d'hygiène au sein des blocs, du financement des activités de gestion des déchets, de la réhabilitation du quartier... Ajouté à ces stratégies proposées par les populations de Mambanda, plusieurs autres solutions idoines peuvent pérenniser et rendrent efficace la gestion des déchets dans ce quartier. Il s'agit:

- de la création, de l'entretien et du renouvellement du réseau d'évacuation des eaux usées (caniveaux, rigoles) pour une meilleure gestion des déchets liquides ;

- de la mise en place d'un dispositif de pré-collecte des ordures ménagères à forte intensité de main-d'œuvre dans les zones peu structurées et à accès difficiles. Cette proposition s'appuie sur le fait que les zones enclavées sont inaccessibles aux camions d'enlèvement des ordures ménagères. En même temps, les distances séparant certains ménages des points de transit sont importantes, d'où la nécessité de la création d'autres points de transit dans chaque coin de ce quartier;

- du perfectionnement de la gestion des déchets en mettant l'accent sur les points sensibles. En effet, la gestion des déchets doit être prise en main et réalisée dans toutes ses étapes par les pouvoirs locaux qui, doivent posséder les équipements, le savoir-faire et le personnel qualifié pour gérer au mieux les rebuts. Ceci doit se faire à travers la valorisation des déchets, le tri de ces déchets à la base, le renforcement de la réglementation qui doit être assortir des sanctions, l'inspection des domiciles des résidents ;

- l'implication des populations à l'élimination des déchets. Une meilleure coopération et une plus grande participation des citoyens, particulièrement au niveau communautaire, peuvent avoir des effets positifs. Ceci est possible grâce à :

- des campagnes d'éducation à l'hygiène et à la gestion des déchets solides qui pourraient être élaborées et mises en œuvre par l'Etat et les administrations locales. Elles seraient financées par le budget bonifié de l'institution chargée de la collecte et de l'élimination des déchets. La population doit être informée sur les modes de collecte, les responsabilités et les avantages du respect des règles d'hygiène. En outre, il faut sensibiliser les populations sur les dangers qui les guettent si elles ne se soucient pas suffisamment de l'élimination des déchets: dégradation de l'environnement, prolifération des mouches, pollution de l'air, propagation des maladies, etc. ;

- l'adoption des divers projets de gestion environnementale par les populations. Des personnalités et des dirigeants communautaires devraient siéger au conseil des organismes responsables de la gestion des déchets. 
- L'adoption des sanctions liées aux mauvaises pratiques de gestion telles que le déversement des ordures dans les lieux publics, les caniveaux et les cours d'eau. Les autorités publiques doivent mettre ces sanctions en pratique et veiller à ce que tout individu qui en déroge soit amandé.

\section{Discussion}

Cette étude est plus axée sur les stratégies pratiques de gestion durable des déchets ménagers. Pourtant au niveau théorique, il existe non seulement des lois et des décrets relatifs à la gestion de ces déchets mais aussi le droit de l'environnement Camerounais.

Les ménages de Mambanda dans leur vécu quotidien produisent d'énormes quantités de déchets. La gestion de déchets ménagers constitue une préoccupation quotidienne à Mambanda. En effet, dans ce quartier de la Commune d'Arrondissement de Douala IV, les ménages accumulent et abandonnent les ordures à proximité des logements, dans les rues, sur des terrains vagues, dans les lieux publics comme les stades ou les marchés, dans les caniveaux à ciel ouvert, dans les cours d'eau, dans les rigoles et drains... Cette situation est similaire à celle vécue à Kinshasa, plus précisément dans la Commune de Limete, et décrite par Likoli (2007). Selon cet auteur, dans presque tous les marchés de cette commune, des monticules d'immondices jonchent les allées des lieux de vente des denrées alimentaires. Cependant, cette situation est contradictoire aux résultats obtenus par Lelo (2008), car selon lui, dans la Commune de Ngaliema, 91\% des ménages possèdent des jardins parcellaires dans lesquels ils enfouissent leurs ordures ménagères.

Les ménages de Mambanda recourent à une kyrielle de méthodes à cause de la faillite du concessionnaire Hysacam qui a la charge de collecter les ordures dans la ville de Douala. En effet, on note qu'à Mambanda, la collecte des ordures par la société Hysacam est très irrégulière. Cette situation est identique à celle vécue par les populations de Bertoua et décrite par Essinga (2013).Cette collecte irrégulière des déchets est due au nombre insuffisant de camions et au mauvais état des rues.

A Mambanda, la situation de l'assainissement est inquiétante. L'environnement est pollué. Ce qui se ressent par la prolifération des maladies liées à l'état de dégradation du cadre de vie des populations. Cette dégradation met quotidiennement en péril la santé et le bien-être des populations. En effet, la présence des eaux usées dans les rues, leur stagnation dans les caniveaux à ciel ouvert encombrés de déchets solides et l'accumulation des eaux pluviales entrainent la dégradation de l'environnement et du cadre de vie, la prolifération des agents pathogènes et accroissent le rythme de contraction des maladies liées à l'environnement. Cette situation est similaire à celle vécue 
dans la ville d'Abidjan et que décrit Pega (2007), et à celle vécue dans la ville de Yaoundé, et mise en relief par Mougoue (2012).

\section{Conclusion}

Cette réflexion met en relief les difficultés de gestion des déchets par les habitants du quartier Mambanda à Douala. Les investigations de terrain montrent que la mauvaise gestion des déchets par les populations locales est à l'origine de la dégradation de l'environnement et induit les problèmes de santé croissants dans ce quartier. Face à ces difficultés, les habitants adoptent des stratégies leur permettant de rendre salubre leur milieu de vie. La société Hysacam et la CUD œuvrent pour la protection de l'environnement à travers de multiples actions d'assainissement. Cependant, ces actions semblent inefficaces face au phénomène d'insalubrité qui ne cesse de prendre de l'ampleur. A cet effet, l'étude propose des stratégies pour l'amélioration de la gestion des déchets dans ce quartier de la Commune d'Arrondissement de Douala IV. Il est primordial d'impliquer les populations à l'élimination des déchets. De ce fait, leurs avis sur les divers projets de gestion environnementale sont pris en compte. L'Etat et les administrations locales doivent organiser des campagnes d'éducation à l'hygiène et à la gestion des déchets solides.

Pour mieux gérer les déchets liquides, les réseaux d'évacuation des eaux usées (caniveaux, rigoles) doivent être bien entretenus et renouvelés. Enfin, il est nécessaire de mettre en place un dispositif de pré-collecte des ordures ménagères à forte intensité de main-d'œuvre dans les zones peu structurées et à accès difficiles. L'instauration des amandes liées à la mauvaise gestion des déchets est primordiale dans le processus d'assainissement du quartier Mambada.

\section{References:}

1. Adamah H., (2001). "La gestion des ordures ménagères dans la ville de Garoua au Cameroun », Mémoire de DEA, FLASH, UAC, 50p.

2. Banque mondiale., (2004). Rapport annuel sur l'efficacité du développement : contribution de la banque mondiale à la réduction de la pauvreté, $134 \mathrm{p}$.

3. Essinga A., (2013). «La gestion des déchets ménagers dans la ville de Bertoua », Université de Yaoundé II- Soa, 87p.

4. Likoli O., (2007). "Niveau de connaissances des habitants de la commune de Limete face à la gestion de leur environnement comme cadre de vie », ISTM Kinshasa, 84p.

5. Lelo N., (2008). Kinshasa Ville environnement. Ed. Harmattan. Paris. $281 \mathrm{p}$. 
6. Mougoué B., Ngnikam E., Feumba R., (2012). «Impacts sanitaires et environnementaux de l'assainissement des eaux usées et des excréta dans les quartiers précaires de Yaoundé (Cameroun)", in Eau et Assainissement, Institut de l'énergie et de l'environnement de la francophonie (IEPF), Numéro 92- 2e trimestre, pp 61-64.

7. Moutila., (2011). "Pression et dynamique de l'espace côtier à mangrove de Youpwe (Douala-Cameroun) », Université de DoualaDEA / Master II, 117p.

8. Ngnikam E., Tanawa E., (2006). Les villes d'Afrique face à leurs déchets. UTBM, Belfort-Montbélliard, $280 \mathrm{p}$.

9. Pega T., (2007). «Assainissement et gestion de l'environnement dans la commune d'Adjame: le cas de Williamsville (Abidjan) », Université de Cocody Abidjan, 140p.

10. Paul A., (2011). "Géomatique et analyse de l'assainissement et des risques sanitaires en milieu urbain: cas du bassin versant de Mbanya dans la ville de Douala (Cameroun) », Université de Ngaoundéré (Cameroun)- Master II géomatique, aménagement et gestion des ressources, $81 \mathrm{p}$.

11. Sane Y., (2002). "La gestion des ordures à Abidjan : Un problème récurrent et apparemment sans solution », in African Journal of Environnemental Assessment and Managment, 13p. 